\title{
Actualización y aproximación clínica a los modelos teóricos de la apraxia de extremidades
}

\author{
Approaching theorical models of limb apraxia: a clinical update
}

Roberta Ghedina, ${ }^{1}$ Patricia Martín-Casas, José Félix Mozo, ${ }^{3}$ Rocío Polanco Fernández ${ }^{4}$

\begin{abstract}
Resumen
La apraxia es una alteración frecuente en las personas que han sufrido un ictus en el hemisferio izquierdo, con importantes repercusiones sobre su calidad de vida. Aunque se han propuesto varios modelos para su interpretación y abordaje, algunos aspectos están aún en estudio. El objetivo de este trabajo es revisar los modelos actuales sobre la apraxia de extremidades, analizar las pruebas de evaluación disponibles y realizar recomendaciones para la práctica clínica. En la revisión se pone de manifiesto que en la literatura existe un mayor interés en el sistema conceptual de la ruta léxico-semántica, sin que haya sido desarrollado en detalle el sistema conceptual de la ruta visuomotora. En este artículo se propone un modelo de las apraxias que supera estas limitaciones y se describe un método de valoración centrado en el conocimiento del cuerpo en la ruta visuomotora. Finalmente se concluye que son necesarios más estudios que validen experimentalmente el modelo propuesto y los métodos de evaluación asociados.
\end{abstract}

Palabras clave: Apraxia, esquema corporal, hemisferio izquierdo, ictus, imitación, valoración

\section{Abstract}

Apraxia is a common disorder in people who have suffered a left hemispheric stroke, with significant impact on their quality of life. Although several models have been proposed for its interpretation and approach, some aspects are still under study. The objective of this study is to review the current models of limb apraxia, to analyze the available evaluation tools and to make recommendations for clinical practice. The review shows that in the literature there is a greater interest in the conceptual system within the lexical-semantic route, without developing in detail the conceptual system within the visuomotor route. This article proposes a model of apraxia that overcomes these limitations and describes an assessment method focused on body knowledge within the visuomotor route. Finally, it is concluded that more studies are necessary to experimentally validate the proposed model and the associated evaluation methods.

Keywords: Apraxia, assessment, body schema, imitation, left hemisphere, stroke

\section{Introducción}

La apraxia es la dificultad o imposibilidad de realizar correctamente movimientos proposicionales aprendidos como consecuencia de una lesión cerebral, en ausencia de trastornos elementales sensoriomotores, alteraciones perceptivas o de comprensión. ${ }^{1}$

Es una alteración que aparece frecuentemente en las personas que han sufrido un ictus y se calcula que al menos el $50 \%$ de ictus cursan con apraxia.2 También puede aparecer en personas que han sufrido otras lesiones cerebrales, tales como un traumatismo craneoencefálico y estadios avanzados en enfermedades neurodegenerativas (por ejemplo, demencia tipo Alzheimer) $)^{3-4}$ La apraxia cursa con mayor frecuencia en aquellos pacientes diestros en los que la lesión está localizada en el hemisferio izquierdo del cerebro. Su prevalencia se estima entre el $28 \%$ y el $57 \%$ en casos con lesiones de hemisferio izquierdo y hasta del $80 \%$ si además cursa con afasia, ${ }^{5}$ mientras que
${ }^{1}$ Facultad de Ciencias de la Salud y Bienestar. Universidad de Vic/Universidad Central de Cataluña. Vic, Cataluña, España.

${ }^{2}$ Sección Departamental de Radiología, Rehabilitación y Fisioterapia, Facultad de Enfermería, Fisioterapia y Podología. Universidad Complutense de Madrid, Madrid, España.

${ }^{3}$ Instituto de Rehabilitación Neurológica, Madrid, España.

${ }^{4}$ Clinica Hildebrand-Centro di riabilitazione Brissago, Suiza.
Correspondencia: José Félix Mozo del Castillo Neuropsicólogo clínico. Instituto de Rehabilitación Neurológica. C/ concejal Francisco José Jiménez Martín 124 - Local, 28047 | MADRID Teléfono: 914631742 / 696092209

E-mail: jose.mozo@irneuro.es 
en lesiones del hemisferio derecho oscila entre el $0 \%$ y el $34 \%{ }^{6-8}$ En demencia tipo Alzheimer su prevalencia se sitúa entre el $35 \%$ y el $98 \%$ de los casos según el estadio en que se encuentre la persona afectada.-10

La apraxia origina una gran discapacidad al repercutir en las actividades y la participación de la persona ${ }^{11-12}$ además de que frecuentemente coexiste con otras alteraciones como la inatención hacia el espacio de la derecha y el deterioro cognitivo. ${ }^{13}$ Es uno de los factores pronósticos de mayor discapacidad y, por ello, origina una gran repercusión en la calidad de vida y un elevado coste derivado de la necesidad de ayuda y adaptaciones que requieren las personas que la padecen.11,14

Tradicionalmente, la apraxia se ha clasificado de varias formas (Tabla 1). En base a su semiología se ha propuesto definirla como apraxia ideativa - que produce la dificultad para la construcción o reconstrucción de la idea del movimiento lo cual impide el uso real de objetos, y su simulación de uso, como apraxia ideomotora - por la que el paciente muestra dificultad en la activación del plan motor - y como apraxia melocinética o cinética - que implica dificultad para realizar movimientos precisos e independientes de los dedos y movimientos rápidos y seriados, conservando la intención.$-^{15}$

Tabla 1. Ejemplos de la clasificación tradicional de la apraxia en función de diferentes factores.

\begin{tabular}{ll}
\hline Factor de clasificación & Denominación \\
\hline Semiología & Ideativa \\
& Ideomotora \\
& Melocinética \\
\hline Actividad afectada & Del vestido \\
& De la marcha \\
\hline Parte del cuerpo & De extremidades superiores \\
& De extremidades inferiores \\
& Bucofacial \\
\hline
\end{tabular}

Además de la clasificación basada en la semiología, se puede contemplar la apraxia en función de la actividad que se ve afectada, como por ejemplo, la apraxia del vestido o la apraxia de la marcha. También se puede clasificar según la parte del cuerpo que se ve afectada, como por ejemplo, la apraxia de las extremidades superiores, la apraxia de las extremidades inferiores o la apraxia bucofacial. En base a estas clasificaciones, su evaluación se ha centrado fundamentalmente en solicitar a la persona que realice gestos o actividades, mediante instrucciones verbales, elicitándolo de forma visual mediante imitación o a través del contacto con un objeto, como reflejan las pruebas descritas a continuación. ${ }^{15}$

A día de hoy, los test con datos normativos en población española son los subtest de la batería Test Barcelona, en concreto los del módulo 3 "Motor y Praxias" que incluye: petición verbal de gestos intransitivos y de uso de objetos, uso secuencial de objetos, imitación de posturas unilateral y bilateral, secuencias de posturas, praxis constructiva gráfica a la orden y copia- y el subtest del Módulo 1 "Lenguaje oral, orientación y atención" - compuesto por praxis orofonatoria no verbal - cuya actualización está disponible desde principios del año 2019! ${ }^{16}$ En la anterior edición del Test Barcelona las pruebas para evaluar la apraxia se encontraban en los subtest del 19 al 24. Otro test diseñado en España y cuya fiabilidad y validez han sido estudiadas es el Evaluation of Upper Limb Apraxia (EULA). ${ }^{17}$

Además de los anteriores, existen otros instrumentos que aunque no estén traducidos y/o validados en España pueden también proporcionar información valiosa para el diagnóstico de apraxia, como el Apraxia Screen of Tulia (AST) que fue construido a partir del Test of Upper Limb Apraxia (TULIA), ${ }^{18-19}$ el Ideomotor Apraxia Test 20 o el Limb Apraxia Battery (LAB) ${ }^{21-22}$ En la mayoría de ellos se solicita verbalmente a través de una orden que el paciente realice una acción familiar, transitiva o intransitiva y también se solicitan mediante imitación acciones con y sin significado.

Existen otros test menos utilizados en España como los alemanes Diagnostic Instrument for Limb Apraxia (DILA), su versión reducida (DILA-S) ${ }^{23}$ y el Köllner Apraxie Screening (KAS), ${ }^{24}$ el italiano Short Test for Ideo-Motor Apraxia (STIMA), ${ }^{25-26}$ el argentino Short Apraxia Screening Test (SAST) ${ }^{27}$ los franceses en la Batería de Evaluación de la Praxia (BEP) y su versión reducida (BREP) ${ }^{28}$ o los australo-americanos de la Florida Apraxia Battery-Extended and Revised Sydney (FABERS) y la Florida Apraxia Battery (FAB) ${ }^{29}$ Todas estas pruebas logran una aproximación al diagnóstico clínico de la apraxia, pero también presentan algunas limitaciones útiles para la práctica clínica, al no valorar el uso de objetos, gestos reales vs no reales, gestos proximales vs distales, transitivos vs intransitivos, reflexivos vs no reflexivos, con significado vs sin significado, problemas mecánicos, secuencias, disociaciones pantomima-uso del objeto, la apraxia voluntaria vs automática, etc.

Si bien estos instrumentos permiten discriminar la presencia de la apraxia y sus características principales, difícilmente permiten entender los procesos subyacentes a la alteración funcional para poder proponer un abordaje terapéutico específico.

Una mejor comprensión de los mecanismos implicados en los gestos y actividades podría facilitar la implementación de un abordaje reeducador, favoreciendo la neuroplasticidad.

No obstante, la evidencia científica actual respalda la compensación como mecanismo preferente de tratamiento de estos pacientes ${ }^{30,31}$ potenciando el uso de estrategias residuales que frecuentemente forman parte de la recuperación espontánea del paciente. 


\section{Objetivos}

Para mejorar la comprensión de los mecanismos práxicos y facilitar su evaluación se propone una revisión narrativa de los modelos actuales sobre la apraxia a partir de los modelos clásicos, para posteriormente detallar algunas de las pruebas de evaluación disponibles. Finalmente se propone un modelo actualizado, que aporta un nuevo mecanismo en la ruta visuomotora y se realizan recomendaciones de valoración aplicables a la práctica clínica.

\section{Modelo clásico de Liepmann}

El término apraxia apareció escrito por primera vez en la obra del lingüista alemán Chaim Heymann Steinthal en 1871,32 quien refiere que los autores contemporáneos utilizaban el término "apraxia" para denominar el trastorno en el uso de objetos y herramientas.

Se atribuye a Hugo Liepmann a principios del siglo XX la primera investigación formal sobre la apraxia, quien la definió como una alteración del movimiento aprendido que se manifestaba con la incapacidad de organizar el movimiento de partes del cuerpo de manera intencional mientras se encontraban preservados los aspectos primarios de la percepción y la producción del movimiento. Por tanto, según este autor la apraxia no es el resultado de la debilidad muscular, la pérdida de sensibilidad, la ataxia, la acinesia, la bradicinesia, la hipometría u otros trastornos del movimiento como el temblor, la distonía, la corea, el balismo, la atetosis o el mioclonus.

Para comprender mejor la apraxia, Liepmann propuso una teoría del control motor que contemplaba dos aspectos distintos y separados de la organización de la acción: la planificación y la producción.

En relación a la planificación Liepmann denominó movement formulae (fórmula del movimiento) o kinetic memories (memorias del movimiento) como formas de memoria espacio-temporal relacionadas a movimientos conocidos y almacenadas en la parte posterior del hemisferio izquierdo (área temporo-parietal) como requisitos previos a la ejecución. ${ }^{15,33}$ Heilmann y cols. en el $1982^{34}$ sugirieron que estas memorias del movimiento están almacenadas sin embargo en el lóbulo parietal inferior izquierdo, en concreto, en el giro supramarginal.

En relación a la producción, estos autores además, denominan el innervatory pattern (patrón de inervación) en referencia al esquema motor correspondiente a una secuencia espacio-temporal que debía trasmitir la fórmula del movimiento a las áreas motoras primarias para la producción del mismo.

El modelo de Liepmann y las aportaciones de Heilmann y cols. pueden explicar por tanto los tres tipos de apraxias de miembro superior anteriormente mencionados:

- La apraxia ideativa que, se produce por pérdida o alteración de la fórmula del movimiento, origina la incapacidad para el uso real de objetos y la pantomima - que es la simulación por medio de gestos y movimientos el uso de un objeto sin utilizarlo realmente -.

- La apraxia ideomotora en la cual, se produce por desconexión de la fórmula del movimiento de los patrones de inervación, la representación espacio-temporal no puede ser codificada en patrones de inervación motores pero el paciente conserva la fórmula del movimiento y mantiene la capacidad de reconocimiento de los gestos. Por ello, el paciente muestra dificultad en la ejecución de gestos precedentemente adquiridos perdiendo la capacidad de su ejecución espontánea y también de su imitación, pero no del reconocimiento.

- La apraxia melocinética o cinética que, se produce por destrucción del patrón de inervación, impide transferir la información al área motora primaria. Esta apraxia se manifiesta con la dificultad para realizar movimientos precisos e independientes de los dedos ${ }^{35}$ y la imposibilidad para realizar movimientos rápidos y seriados, tales como presionar un botón, tocar el piano, etc. Además, se observa una pérdida de velocidad y exactitud en el movimiento, sin embargo la intención quedaría preservada ${ }^{36}$

Los tres tipos de apraxia se manifiestan en ambas extremidades por lesión hemisférica izquierda.

\section{Sistema conceptual y sistema de producción}

A principios de los 80 el equipo canadiense encabezado por Eric A. Roy ${ }^{37}$ propuso un modelo de praxias en el cual se distinguen dos sistemas: uno conceptual y uno de producción, cuyo planteamiento se ha reforzado con los años. ${ }^{38}$

En 1991 el grupo americano de Florida, ${ }^{1}$ creó un nuevo modelo que actualiza estos sistemas y los relaciona con dos rutas de procesamiento cognitivo: la ruta léxicosemántica y la ruta visuomotora. Se trata de un modelo inspirado en el lenguaje que surgió desde la neurociencia cognitiva.

El sistema conceptual es aquel que ofrece al sujeto el significado de los gestos, y garantiza su reconocimiento y/o identificación en su correspondiente contexto. Los autores sustituyeron el concepto de fórmula del movimiento descrito por anteriores autores ${ }^{33-37}$ por el de lexicón gestual y lo dividieron en dos módulos, uno de entrada y uno de salida, al igual que existía en el modelo contemporáneo del lenguaje ${ }^{39-40}$ Los lexicones anteriormente descritos pertenecen a la ruta léxico-semántica donde el lexicón de entrada sirve para el reconocimiento de los gestos familiares y el lexicón de salida facilita la producción de los gestos ejecutados previamente.

El sistema de producción es aquel que permite la sa- 
lida del gesto, no obstante cuando el gesto es desconocido para el sujeto, no se precisa de acceso a los léxicones del sistema conceptual. Esta ruta conocida como visuomotora permite la transformación del gesto observado en acto motor sin beneficiarse del sistema conceptual.

\section{La imitación en la apraxia}

En 1962, el neurólogo americano Norman Geschwind y la psicóloga Edith Kaplan observaron a un paciente que podía realizar la pantomima por orden verbal con la mano derecha mientras presentaba dificultades con la izquierda. Sin embargo, con ambas manos podía imitar los gestos y usar los objetos correctamente. Siguiendo el esquema del lenguaje elaborado por Wernicke a finales del siglo XIX, los autores interpretaron que la disociación entre la mano izquierda y la derecha a la orden verbal eran debidos a una desconexión del córtex motor derecho y el córtex motor izquierdo en el área del lenguaje y atribuyeron la causa a una lesión del cuerpo calloso. Por tanto, una lesión del cuerpo calloso produciría apraxia sólo en el miembro superior izquierdo. ${ }^{41}$

En 1975 Geschwind propuso un modelo neo-asociacionista en el cual explicó la fórmula del movimiento de Liepmann desde la comprensión verbal y la apraxia, según él, sería el resultado de una desconexión entre el área de Wernicke y las áreas de asociación motoras derechas e izquierdas por la interrupción del fascículo arqueado o por la lesión del giro supramarginal izquierdo.

Según este autor sólo el gesto producido a partir de una orden verbal requiere los procesos del hemisferio izquierdo. No obstante, como en aquella época se describieron también casos de pacientes apráxicos que no podían imitar, para justificar los fallos en la imitación Geschwind sugirió que el fascículo arqueado resulta también decisivo para la transformación visuomotora. ${ }^{42}$

Si bien este modelo explica los errores de los pacientes en la imitación y la pantomima por orden verbal, no ayuda a comprender las dificultades en el uso de objetos que pueden presentar también los pacientes con apraxia.

En 1980 el neurólogo italiano Ennio De Renzi, profundizó en la apraxia por imitación y creó un test de imitación para evaluarla. En esta prueba, excluyó la modalidad auditiva (orden verbal) para valorar la producción del gesto, siendo de esta manera fácilmente suministrable a pacientes afásicos. ${ }^{8}$ Así, cuando el examinador pide la producción del gesto a través de la imitación está facilitando al paciente el acceso a la fórmula del movimiento y se valora la capacidad del paciente para transformar la secuencia espacio-temporal en gesto. ${ }^{43}$

Este test ${ }^{8}$ es un test de imitación que incluye gestos intransitivos divididos en tres categorías: a) gestos familiares con significado y gestos no familiares (nuevos), b) gestos que incluyen segmentos proximales y distales de la extremidad superior y c) posiciones y secuencias de gestos. No incluye la utilización de objetos reales, ni la petición de pantomima. Esta última se considera la valoración más sensible para detectar alteraciones de la fórmula del movimiento; sin embargo, las dificultades de comprensión verbal de la tarea sobre todo en pacientes graves, obliga a utilizar una prueba de imitación.

En este sentido, el test propuesto por De Renzi, valora principalmente el sistema de producción y menos el sistema conceptual contribuyendo a la detección de la apraxia ideomotora. Desde el punto de vista clínico y teórico, una mejor imitación en gestos familiares respecto a gestos nuevos podría implicar la conservación de la fórmula del movimiento. Hay que considerar también que, a veces sucede que los gestos nuevos, no familiares, sean lexicalizados por lo que son reproducidos recuperando información familiar (por ejemplo, si se pide la imitación del gesto "mano horizontal en la frente", el paciente podría recuperar el gesto del saludo militar y adecuarlo a lo que está observando). De hecho, entre los años 70 y 90 del siglo pasado varios autores describieron pacientes que a pesar de no reconocer la familiaridad del gesto consiguen reproducirlo por imitación. Otros pacientes acceden al significado del gesto y a su aplicación contextual, pero fallan en todas las tareas de ejecución, solicitadas tanto por imitación como por orden verbal. ${ }^{1,5,44-45}$

\section{Modelo contemporáneo}

En el año 2000, Cubelli y colaboradores ${ }^{46}$ modificaron el modelo anterior, ${ }^{1}$ realizando las siguientes aportaciones:

1. Sustituyen el módulo de esquemas motores de inervación ${ }^{1,33,47}$ por el almacén a corto plazo motor (buffer). Un daño en el almacén a corto plazo tendría que afectar más a la secuencia de gestos que a gestos aislados como las posturas. ${ }^{48}$

2. Introducen el concepto de esquema corporal. La alteración del esquema corporal es frecuente en pacientes que además de presentar dificultad en la imitación de gestos nuevos, por lesión en la vía directa o visuomotora, encuentran dificultad también en reproducir los mismos gestos en un maniquí. ${ }^{49}$

3. Suprimen la vía que une los dos lexicones (ver modelo adaptado Rothi, Ochipa, Heilman).

La falta de evidencia de trastornos en la comprensión para la ejecución de gestos familiares ha llevado a eliminar la conexión directa entre el lexicón de entrada y el de salida, dado que para la producción de gestos familiares parece exigir el acceso a la semántica. ${ }^{46}$

\section{Actualización en la evaluación de la apraxia} En 2008 los italianos Angela Bartolo, Roberto $\mathrm{Cu}-$ 
belli y Sergio della Sala presentaron una nueva batería para la valoración de la apraxia: la Limb Apraxia Battery $(\mathrm{LAB})^{21-22}$ Se trata de una batería capaz de valorar los módulos descritos en el modelo cognitivo de las praxias de Cubelli y cols. ${ }^{22,46}$ La batería divide las pruebas en dos sistemas: el sistema de recepción (hasta ahora definido como sistema conceptual) y el sistema de producción.

El sistema de recepción contempla dos niveles de procesamiento que corresponden al módulo del lexicón de entrada y al módulo de la memoria semántica de la acción valorados respectivamente a través de tareas de reconocimiento e identificación. Los gestos utilizados para dichas tareas son de dos tipos: intransitivos y pantomimas. La novedad de esta batería es la inclusión de pruebas que no requieren la producción del gesto, lo que permite valorar el proceso de elaboración previo a la producción, concretamente la fórmula del movimiento citada por Liepmann.

El sistema de producción contempla las dos rutas, la vía indirecta o léxico-semántica y la vía directa o de conversión visuomotora. La primera se valora a través de pruebas por orden verbal, presentación de estímulos visuales (viñetas de contexto a emparejar con el gesto mostrado por el examinador) y visuotáctiles con uso real del objeto. Esta batería evalúa la producción de los siguientes tipos de gestos:

a. Los gestos intransitivos. Estos pueden solicitarse a partir de estímulos visuales, mediante un dibujo de una escena con contexto asociado al gesto. Por ejemplo, un soldado delante de un general para solicitar el gesto del saludo militar, de modo que pueda valorarse su producción en pacientes afásicos.

b. La pantomima. Esta simula el uso de un objeto como si estuviera en la mano del paciente, que tiene que considerar la función del objeto y también la configuración de los dedos de la mano y su orientación respecto al objeto, así como la distancia del objeto al destinatario de la acción.

c. Los gestos transitivos. Estos incluyen una tarea en la que el examinador usa un objeto delante del paciente y el paciente lo imita posteriormente. Los gestos transitivos requieren el uso concreto del objeto, hecho que podría facilitar la producción del gesto mediado por la visión y la manipulación directa. La posibilidad de que el examinador muestre el uso del objeto antes de que el paciente lo use ofrece al paciente no solo el estímulo del objeto, sino también el esquema espacio-temporal del gesto;

d. Los gestos no familiares. Estos, no pertenecen al conocimiento adquirido del paciente, se solicitan solo por imitación.

En el año 2011 Alessia Tessari, Alessio Toraldo, Alberta Lunardelli, Antonietta Zadini y Raffaella Ida Rumia- ti presentaron en Italia una nueva prueba estandarizada de imitación de gestos intransitivos para el diagnóstico de la apraxia ideomotora. ${ }^{26}$ Además de asignar un valor global de diagnóstico, este test valora la posible disociación entre gestos familiares vs no familiares, entre efector proximal vs distal y entre vía léxico-semántica vs vía directa o de conversión visuomotora.

Este test pone énfasis en la presentación por separado de los gestos familiares y no familiares. Se ha demostrado que la presentación de los gestos por parte del examinador en listas separadas permite un mejor diagnóstico de la disociación de las vías. Los autores enseñan a suministrar en orden riguroso primero la lista de gestos familiares y luego la de gestos no familiares para evitar la activación exclusiva de la vía directa o de conversión visuomotora para ambos gestos.

La vía directa es más coste-beneficiosa porque permite la imitación tanto de gestos no familiares como de gestos familiares. La presentación de los estímulos por separado pone de manifiesto el estado de cada una de las vías, sin compensación entre ellas. Cuando las listas son mixtas el paciente tiene que activar una y otra, poniendo en juego mayor flexibilidad cognitiva. Sin embargo, la vía directa que necesita un análisis más detallado de la configuración espacio-temporal del gesto observado y, por tanto, mayor carga de la memoria de trabajo, resuelve la tarea para ambos gestos eludiendo el acceso a su significado. De este modo, el paciente imita los gestos familiares como si fueran desconocidos. El uso de la vía directa es una estrategia común de compensación cognitiva en el sujeto sano en situación de estrés y en pacientes con daño cerebral adquirido del hemisferio izquierdo. ${ }^{50}$

Ninguna de las pruebas anteriores está actualmente validada en España.

\section{Implicaciones clínicas para la valoración de los pacientes apráxicos}

A partir de los modelos revisados para la interpretación de la apraxia se puede concluir que la función práxica es una función de alto nivel de complejidad y que requiere la integración de varios procesos cognitivos: perceptivos, mnésicos y ejecutivos entre otros. En función del tipo de disociaciones descritas, se puede afirmar que la función pertenece a un sistema multi-dominio y modular. ${ }^{15,51}$ Por la naturaleza misma de este sistema los errores práxicos observados han sido difícilmente clasificables o parcialmente comprensibles en el pasado; actualmente se dispone de instrumentos de valoración de la apraxia que son aplicables incluso en pacientes afásicos e incluyen la separación entre gesto familiar vs no familiar, gesto transitivo vs intransitivo, pantomimas en reconocimiento vs producción a partir de orden verbal, estímulo visual vs táctil-cinestésico vs auditivo y proximal vs distal. Asimismo, distinguir estas disociaciones facilita la rehabilitación de la apraxia 
(por ejemplo, distinguir en segmentos corporales proximales y distales, facilita la localización precisa de la parte del cuerpo a tomar en cuenta durante la intervención).22,26

A pesar de que existe numerosa literatura al respecto, son escasas las aportaciones que evalúan el significado del error revelado, especialmente en la imitación de gestos no familiares. Cuando se valora la capacidad del paciente de imitar gestos, en particular, los no conocidos, se evalúa en realidad su capacidad para resolver un problema respecto a su propio cuerpo. Este tipo de problema requiere un conocimiento que desvela "cómo usar el cuerpo" para lograr una cierta posición de la extremidad valorada. Para la resolución del problema el paciente utiliza la observación de los segmentos que el examinador mueve (“¿Qué mueve? ¿Cómo hace para moverlo?”) y la transformación de esos mismos en coordenadas egocéntricas. En definitiva, usa el conocimiento del cuerpo para resolver un problema de tipo mecánico y se supone que para ello el paciente activa la representación de su propio cuerpo, es decir el esquema corporal.

Los autores de este artículo concuerdan con autores como Salimi Dafsari y cols. $(2019)^{52}$ en la necesidad de activar en una fase inicial de la programación del gesto el Sistema de Descripción Estructural del Cuerpo (SDEC), es decir, el mapa topológico que define las partes del cuerpo, su extensión y sus relaciones espaciales. El SDEC se puede equiparar al Sistema de Descripción Estructural del Objeto (SDEO), es decir, a las características físicas del objeto manipulable. Además del SDEC sería necesario activar el Sistema Semántico Corporal (SSC), que es una representación léxico-semántica del cuerpo que incluye los nombres de las partes del cuerpo, su función y su relación con su entorno. ${ }^{52} \mathrm{El}$ SSC es fundamental en su sub-componente funcional, que hemos denominado: Sistema Semántico Corporal funcional (SSCf), que es una representación del cuerpo que incluye el rol articular y las determinadas posibilidades cinemáticas de cada una de las articulaciones humanas y que determinan el movimiento biológico.

El paciente apráxico ideomotor podría cometer errores cinemáticos en la acción porque, independientemente del significado o del contexto del gesto, podría encontrar dificultad en la manipulación de su propio cuerpo como fue demostrado en la manipulación de un cuerpo externo o maniquí por Goldenberg (1995)..$^{49}$ Tomando en consideración esta aportación, proponemos un método de valoración del SSCf que consiste en primer lugar en acordar con el paciente una postura final usando una imagen en 2D o 3D de un maniquí o una persona adoptando una postura concreta. En segundo lugar, el examinador realiza una movilización pasiva en el miembro superior ipsilateral del paciente para el logro de esa postura esperada, y si se revela una resistencia para el logro de esa postura, podría indicarnos que el paciente no ha creado una expectativa de su propio movimiento y por tanto podría indicar una difi- cultad de acceso al esquema corporal, en su sub-componente SSCf. Esta resistencia se detecta también en el lado contralateral a la lesión hemisférica y no debe confundirse con la respuesta al estiramiento del músculo presente en el miembro hipertónico.

A continuación, se presenta un modelo que integra los conceptos anteriormente revisados y que permite una nueva aproximación en la valoración del paciente apráxico (Figura 1). Se han considerado los conceptos referidos en Salimi Dafsari (2019) $)^{52}$ junto a las aportaciones ya expuestas de Rothi, Ochipa y Heilman (1991), Rumiati y Tessari $(2002)^{48}$ y Cubelli y cols. (2000).$^{46}$ En conclusión, la evolución en la comprensión y evaluación de la apraxia de extremidades ha sido muy relevante en los últimos años, motivando el desarrollo de nuevas escalas de evaluación del paciente apráxico cuya validación en España es recomendable. No obstante, se recomienda la inclusión del SSCf en los futuros modelos tanto de evaluación como del abordaje clínico.

Además, se requiere la realización de estudios que demuestren la relevancia del esquema corporal en la interpretación de la apraxia de extremidades para facilitar la aplicación y evaluación de estrategias terapéuticas basadas en la mejora de la representación del esquema corporal y de la acción.

\section{Discusión}

El objetivo de esta revisión era mejorar la comprensión de los mecanismos práxicos a partir de la descripción de las aportaciones de los modelos clásicos y de la aportación que en este texto se hace sobre un nuevo mecanismo del sistema conceptual en la ruta directa o de conversión visuomotora, valorable a partir de la movilización pasiva del paciente dado un objetivo de movimiento acordado a alcanzar.

La idea de esta revisión nace a partir de la inspiración tras la lectura e investigaciones de Georg Goldenberg en $1995^{32}$ a partir de sus estudios realizados en tareas experimentales con la manipulación de maniquís, resaltando la importancia de la participación del esquema corporal a nivel conceptual.

A partir de ahí se han revisado de forma histórica los modelos de la apraxia y hemos planteado una actualización de los modelos actuales incorporando evidencia de la literatura y de la práctica clínica.

El texto ha puesto el foco de atención en la ruta visuomotora, principalmente, dado su compromiso con el esquema corporal, dejando de lado nuevas propuestas para la ruta léxico-semántica.

Dadas limitaciones en la extensión del artículo y en el contenido teórico a revisar se ha desgranado la ruta visuomotora en subcomponentes del esquema corporal (véase en Figura 1 SDEO, SDEC o SSC) como se había realizado con anterioridad por otros autores siguiendo el paradigma 
estudiado a partir del sistema conceptual y los lexicones, y además se ha incorporado al modelo una nueva aportación referida al SSCf.

Finalmente, en base a este modelo actualizado (véase Figura 1) se ha hecho una propuesta clínica de valoración donde el terapeuta propone al paciente la observación de una postura a alcanzar (bien sea en 2D o 3D) y posteriormente se realiza una movilización pasiva en el miembro superior ipsilateral a la lesión con el objetivo de posicionarlo en la postura observada.

En relación a las limitaciones de este estudio, esta revisión ha seguido una metodología narrativa lo que implica que se ha podido obviar algunos artículos de interés para el tema así como incluir algunos de calidad científica baja.

Se espera que a partir de este trabajo se puedan superar las limitaciones anteriormente expuestas llevando a cabo una metodología sistemática de revisión, una refutación del modelo a través de paradigmas experimentales, y una mayor protocolización y estandarización del procedimiento asociado de valoración.

Como punto final cabe destacar que el contenido de este trabajo se ha realizado por profesionales en contacto directo con laborales asistenciales y académicas de forma simultánea, lo cual se considera que aporta un valor añadido al enfoque de la revisión, las apreciaciones clínicas hechas y al desarrollo a lo largo del texto.

\section{Referencias}

1. Gonzalez Rothi LJ, Ochipa C, Heilman KM. A cognitive neuropsychological model of limb Praxis. Cogn Neuropsychol. 1991;8(6):443-58. https://doi. org/10.1080/02643299108253382

2. Bickerton W-L, Riddoch MJ, Samson D, Balani AB, Mistry B, Humphreys GW. Systematic assessment of apraxia and functional predictions from the Birmingham Cognitive Screen. J Neurol Neurosurg Psychiatry. 2012;83(5):513-21. http://dx.doi. org/10.1136/jnnp-2011-300968

3. Derouesné C, Lagha-Pierucci S, Thibault S, Baudouin-Madec V, Lacomblez L. Apraxic disturbances in patients with mild to moderate Alzheimer's disease. Neuropsychologia. 2000;38(13):1760-9. https://oi.org/10.1016/S0028-3932(00)00081-6

4. Stamenova V, Roy EA, Black SE. A model-based approach to understanding apraxia in Corticobasal Syndrome. Neuropsychol Rev. 2009;19(1):47-63. https://doi.org/10.1007/s11065-008-9079-5

5. De Renzi E, Pieczuro A, Vignolo LA. Ideational apraxia: A quantitative study. Neuropsychologia. 1968;6(1):41-52. https://doi.org/10.1016/00283932(68)90037-7

6. Donkervoort M, Dekker J, van den Ende E, Stehmann-Saris JC, Deelman BG. Prevalence of apraxia among patients with a first left hemis-

Figura 1. Modelo de apraxia revisado.

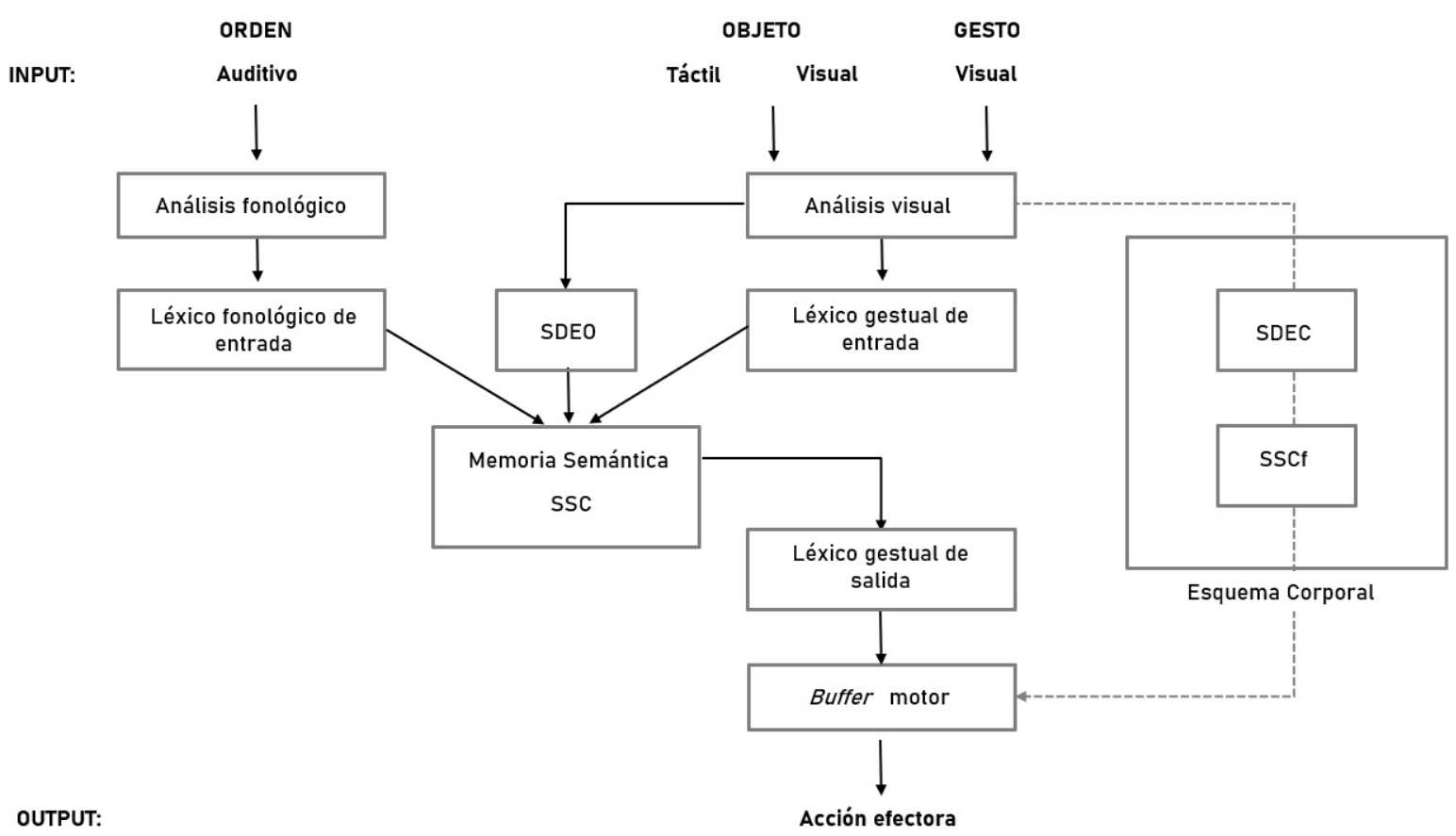

En líneas discontinuas se encuentra la ruta de conversión visuo-motora que permite reproducir el gesto nuevo y en líneas continuas la ruta léxico-semántica que permite la reproducción del gesto conocido. SDEO = Sistema de Descripción Estructural del Objeto; SDEC = Sistema de Descripción Estructural del Cuerpo; SSC = Sistema Semántico Corporal; SSCf = Sistema Semántico Corporal funcional 
phere stroke in rehabilitation centres and nursing homes. Clin Rehabil. 2000;14(2):130-6. https://doi. org/10.1191/026921500668935800

7. Dovern A, Fink GR, Weiss PH. Diagnosis and treatment of upper limb apraxia. J Neurol. 2012;259(7):1269-83. https://doi.org/10.1007/ s00415-011-6336-y

8. De Renzi E, Motti F, Nichelli P. Imitating gestures. A quantitative approach to ideomotor apraxia. Arch Neurol. 1980;37(1):6-10. https://doi.org/10.1001/ archneur.1980.00500500036003

9. Smits LL, Flapper M, Sistermans N, Pijnenburg YAL, Scheltens P, van der Flier WM. Apraxia in mild cognitive impairment and Alzheimer's disease: validity and reliability of the Van Heugten test for apraxia. Dement Geriatr Cogn Disord. 2014;38(12):55-64. https://doi.org/10.1159/000358168

10. Edwards DF, Deuel RK, Baum CM, Morris JC. A quantitative analysis of apraxia in senile dementia of the Alzheimer type: Stage-related differences in prevalence and type. Dement Geriatr Cogn Disord. 1991;2(3):142-9. https://doi.org/10.1159/000107189.

11. Bjørneby ER, Reinvang IR. Acquiring and maintaining self-care skills after stroke. The predictive value of apraxia. Scand J Rehabil Med. 1985;17(2):75-80. PMID: 4023662

12. Wu AJ, Burgard E, Radel J. Inpatient rehabilitation outcomes of patients with apraxia after stroke. Top Stroke Rehabil. 2014;21(3):211-9. https://doi. org/10.1310/tsr2103-211

13. Wee JYM, Hopman WM. Stroke impairment predictors of discharge function, length of stay, and discharge destination in stroke rehabilitation. Am J Phys Med Rehabil. 2005;84(8):604-12. https://doi. org/10.1097/01.phm.0000171005.08744.ab.

14. Foundas AL, Macauley BL, Raymer AM, Maher LM, Heilman KM, Rothi LJG. Ecological implications of limb apraxia: Evidence from mealtime behavior. J Int Neuropsychol Soc. 1995;1(1):62-6. https://doi.org/10.1017/S1355617700000114

15. Ustárroz JM, Ríos Lago M, F MU. Manual de neuropsicología. Barcelona: Viguera; 2008.

16. Peña-Casanova, J. Test Barcelona - Estación de trabajo. 2019. https://www.test-barcelona.com/es/

17. Pérez Mármol JM, López Alcalde S, Carnero Pardo C, Cañadas de la Fuente GA, Peralta Ramírez MI, García Ríos M del C. Creación y diseño de un test para la evaluación de la apraxia de los miembros superiores (EULA) basado en un modelo cognitivo: un estudio piloto. Rev Neurol. 2015;60(02):66. MEDES: 95850. https://doi.org/10.33588/rn.6002.2013575

18. Vanbellingen T, Kersten B, Van de Winckel A, Bellion M, Baronti F, Muri R, et al. A new bedside test of gestures in stroke: the apraxia screen of TULIA (AST). J Neurol Neurosurg Psychiatry. 2011;82(4):389-92. http://dx.doi.org/10.1136/jnnp.2010.213371.
19. Vanbellingen T, Kersten B, Van Hemelrijk B, Van de Winckel A, Bertschi M, Müri R, et al. Comprehensive assessment of gesture production: a new test of upper limb apraxia (TULIA): A new test of upper limb apraxia. Eur J Neurol. 2010;17(1):59-66. https://doi.org/10.1111/j.1468-1331.2009.02741.x

20. Alexander MP, Baker E, Naeser MA, Kaplan E, Palumbo C. Neuropsychological and neuroanatomical dimensions of ideomotor apraxia. Brain. 1992;115(1):87-107. https://doi.org/10.1093/brain/115.1.87

21. Bartolo A, Drei S, Cubelli R, Lab DSS. Limb Apraxia Battery. Firenze: Giunti; 2008.

22. Bartolo A, Cubelli R, Della Sala S. Cognitive approach to the assessment of limb apraxia. Clin Neuropsychol. 2008;22(1):27-45. https://doi. org/10.1080/13854040601139310

23. Randerath J, Buchmann I, Liepert J, Büsching I. Diagnostic instrument for limb apraxia : Short version (DILA-S). 2017 [citado el 03 de septiembre de 2019]; Disponible en: http://nbn-resolving.de/ urn:nbn:de:bsz:352-2-luq46vs8q2f0i8

24. Weiss PH, Kalbe E, Kessler J, Fink GR, Binder E, Hesse MD, et al. Kölner apraxie screening. Göttingen, Germany: Hogrefe; 2013.

25. Tessari A, Toraldo A, Lunardelli A, Zadini A, Rumiati RI. STIMA: a short screening test for ideo-motor apraxia, selective for action meaning and bodily district. Neurol Sci. 2015;36(6):977-84. https://doi.org/10.1007\%2Fs10072-015-2203-4.

26. Lunardelli A, Rumiati RI, Tessari A, Toraldo A, Zadini A. Prova standardizzata per la diagnosi del disturbo aprassico ideomotorio selettivo per tipo di gesto e tipo di effettore. Ric psicol [Internet]. [citado el 5 de septiembre de 2019]; Disponible en: https:// www.medra.org/servlet/MREngine?hdl=10.3280/ RIP2011-003001

27. Leiguarda R, Clarens F, Amengual A, Drucaroff L, Hallett M. Short apraxia screening test. J Clin Exp Neuropsychol. 2014;36(8):867-74. https://doi.org/10. 1080/13803395.2014.951315

28. Peigneux P, Van der Linden M. Présentation d'une batterie neuropsychologique et cognitive pour l'évaluation de l'apraxie gestuelle. Rev neuropsychol [Internet]. 2000 [citado el 15 de septiembre de 2019];10(2). Disponible en: https://orbi.uliege.be/ handle/2268/241138.

29. Power E, Code C, Croot K, Sheard C, Gonzalez Rothi LJ. Florida Apraxia Battery-Extended and revised Sydney (FABERS): design, description, and a healthy control sample. J Clin Exp Neuropsychol. 2010;32(1):1-18. https://doi. org/10.1080/13803390902791646

30. MAGIC Evidence Ecosystem Foundation. MAGICapp. Magicapp.org. 2019. https://app.magicapp. org/\#/guideline/4473

31. Ramos-Galarza, C; Ramos, V; Jadán-Guerrero, J; 
Lepez-Martínez, N; Gómez-García, A; BolañosPasquel, M. Conceptos Fundamentales en La Teoría Neuropsicológica. Revista Ecuatoriana de Neurología. 26.1. 2017: 53-60. http://scielo.senescyt. gob.ec/scielo.php?script=sci_arttext\&pid=S263125812017000300053\&lng=es

32. Goldenberg G. Apraxia - the cognitive side of motor control. Cortex. 2014;57:270-4. https://doi. org/10.1016/j.cortex.2013.07.016

33. Liepmann $\mathrm{H}$. The left hemisphere and action. London, Ontario: University of Western Ontario; 1905.

34. Heilman KM, Rothi LJ, Valenstein E. Two forms of ideomotor apraxia. Neurology. 1982;32(4):342-6. https://doi.org/10.1212/WNL.32.4.342

35. Heilman KM, Rothi LJG. Limb apraxia: A look back. Apraxia: The neuropsychology of action. 1997;7-18.

36. Ardila A. Agnosias y Apraxias. Revista Neuro Psicología, Neuropsiquiatría y Neurociencias. 2015;15, No 1.

37. Roy EA, Square PA. Common considerations in the study of limb, verbal and oral apraxia. En: Advances in Psychology. Elsevier; 1985. p. 111-61. https://doi. org/10.1016/S0166-4115(08)61139-5

38. Stamenova V, Black SE, Roy EA. An update on the Conceptual-Production Systems model of apraxia: evidence from stroke. Brain Cogn. 2012;80(1):5363. https://doi.org/10.1016/j.bandc.2012.03.009

39. Patterson, Karalyn, et al. Speak and spell: Dissociations and word-class effects. En: The Cognitive Neuropsychology of Language (Psychology Revivals). Psychology Press; 2013. p. 289-310.

40. Ellis AW, Young AW, Anderson C. Modes of word recognition in the left and right cerebral hemispheres. Brain Lang. 1988;35(2):254-73. https://doi. org/10.1016/0093-934X(88)90111-3

41. Geschwind N. Disconnexion syndromes in animales and man. Brain. 1965;88(2):237-237.

42. Geschwind N. The apraxias: neural mechanisms of disorders of learned movement. Am Sci. 1975;63(2):188-95.

43. de Renzi E, Faglioni P, Sorgato P. Modality-specific and supramodal mechanisms of apraxia. Brain. 1982;105(2):301-12. https://doi.org/10.1093/ brain/105.2.301

44. Rothi LJ, Heilman KM, Watson RT. Pantomime comprehension and ideomotor apraxia. J Neurol Neurosurg Psychiatry. 1985;48(3):207-10. http:// dx.doi.org/10.1136/jnnp.48.3.207

45. Heilman KM, Rothi LG, Mack L, Feinberg T, Watson RT. Apraxia after a superior parietal lesion. Cortex. 1986;22(1):141-50. https://doi.org/10.1016/ S0010-9452(86)80038-7

46. Cubelli R, Marchetti C, Boscolo G, Della Sala S. Cognition in action: testing a model of limb apraxia. Brain Cogn. 2000;44(2):144-65. https://doi. org/10.1006/brcg.2000.1226

47. 47. Rothi, LJ Gonzalez, Cynthia Ochipa, and Ken- neth M. Heilman. A cognitive neuropsychological model of limb praxis and apraxia. Apraxia: The neuropsychology of action. 1997: 29-49. https://doi. org $/ 10.1080 / 02643299108253382$

48. Rumiati RI, Tessari A. Imitation of novel and wellknown actions: The role of short-term memory. Exp Brain Res. 2002;143(3):395-395. https://doi. org/1007/s00221-001-0956-x

49. Goldenberg G. Imitating gestures and manipulating a mannikin--the representation of the human body in ideomotor apraxia. Neuropsychologia. 1995;33(1):63-72. https://doi. org/10.1016/0028-3932(94)00104-W

50. Cubelli R, Bartolo A, Nichelli P, Della Sala S. List effect in apraxia assessment. Neurosci Lett. 2006;407(2):118-20. https://doi.org/10.1016/j. neulet.2006.08.019

51. 51. Silva-Barragán, M; Ramos-Galarza, C. Modelos de Organización Cerebral: un recorrido neuropsicológico. Revista Ecuatoriana de Neurología. 29.3. 2020: 74-83. https://doi.org/10.46997/revecuatneurol29300074

52. Dafsari HS, Dovern A, Fink GR, Weiss PH. Deficient body structural description contributes to apraxic end-position errors in imitation. Neuropsychologia. 2019;133(107150):107150. https://doi. org/10.1016/j.neuropsychologia.2019.107150

Conflictos de intereses y fuentes de financiación: Ninguno de los autores mantiene ninguna relación con personas $u$ organizaciones que pudiesen influenciar de forma inapropiada su trabajo. Este estudio no recibió financiación externa. 